\title{
SIMULATION OF THE ADMIXTURE SPREADING IN A STEADY 2D LENGTHY STREAM OF A VISCOUS FLUID
}

\author{
A.V. BABAYAN* \\ K.A. NADOLIN
}

Received: 22/11/99

Accepted: 24/03/00

\author{
Department of Mechanics and Mathematics, Rostov State University, \\ 344090, Zorge st, 5, Rostov-on-Don, Russia \\ artem_vb@math.rsu.su,nadolin@math.rsu.ru
}

*to whom all correspondence should be addressed

\begin{abstract}
The problem of the passive contaminant spreading in a steady viscous fluid stream is discussed while the admixture's dissipation and diffusion are taken into account. The channel is assumed to be a horizontal plane, curvilinear and quite lengthy, so that the ratio of the stream width to its length can be regarded as a small parameter. A mathematical model of the process derived by the small parameter technique from the 2D steady Navier-Stokes equations for incompressible viscous fluid and non-steady convection-diffusion equation of a substance in the moving medium is introduced. A finite element method is applied for numerical study of the proposed model and results of computer experiments are presented.
\end{abstract}

\section{INTRODUCTION}

The mathematical models of the contaminant spreading in the natural streams are an important part of the water ecology simulation (Chatwin and Allen, 1985). They are widely used for the surface water quality control. For simulations of regional scale, the so-called 'camera' models are widely used. The region is divided into relatively homogenous sub-regions (cameras); characteristics are averaged and considered to be constant inside each camera. The boundary balance relations describes the interaction among the cameras. However, in some cases the intracamera processes are of interest and this requires the application of more detailed 'distributed' models (Chatwin and Allen, 1985).

Usually in water ecology problems these 'distributed' models are restricted to consider the passive admixture, i.e. varying of the contaminant concentration is assumed not to affect the hydrody- namic part of the model - velocity and pressure fields. The approach allows to split the problem in two; the first one is to determine the flow velocity field and the second problem is to obtain the admixture concentration in a flow with this known velocity field.

One peculiarity of the river ecosystems is the considerable length of the channel and its relatively small width. The theoretical analysis of the admixture spreading in the lengthy stream of viscous fluid was initiated by Taylor $(1953,1954)$ and Aris (1956). The term 'Taylor dispersion' is now being widely used in literature, to identify this problem (Monin and Yaglom, 1979; Kotorynski 1994; Rosencrans 1997).

In the modelling of the contaminant propagation in the viscous flow, it is necessary to solve the Navier-Stokes and the convection-diffusion equations in a coupled manner. Because of the complexity and the non-linear character of this system, 
the reduced models of the admixture spreading are of special interest (Chatwin and Allen, 1985; Rosencrans, 1997). Usually the velocity fields in the reduced models are determined on the basis of experimental data, semi-empirical equations, averaged-value or analytical representations. When constructing the reduced models of admixture spreading in reservoirs, it is usual to average the concentration field in one or two spatial variables. Because of the small ratio of the channel stream width to its length, it is possible to apply various small parameter techniques (Babayan, 1997; Fife, 1975; Kotorynski, 1994; Nadolin, 1997). This approach allows reducing the original system of differential equations by excluding terms, which are not essential for flows of this shape. Usually, equations of the first approximations take into account only longitudal convection and diffusion. This is justified when the transversal diffusion is not significant. Nevertheless, in some cases (in river streams, for instance) it is not true (Fischer, 1969; Yotsukura and Sayre, 1976).

With the current work, we introduce the model of the non-conservative passive contaminant spreading in 2D lengthy stream channel, using the technique of Nadolin, Botchev and Nicolayev (1997). The starting point of the derivation is the NavierStokes equations for the 2D stationary incompressible viscous flow and the time-dependent diffusion equation of a substance in the moving average. As a result, for each unknown parameter, it gives an initial boundary-value problem in terms of the unknown's expansion with respect to the small parameter - the ratio of the stream width to its length. Since the admixture is assumed to be passive, we calculate the unknown velocity and the pressure fields. The remaining equation for the concentration has to be solved numerically. The particular feature of the model is taking into account the stream cross-structure that allows studying the contaminant spreading in a channel with varying width.

\section{PROBLEM FORMULATION}

Consider a non-conservative admixture spreading in a 2D steady stream with known boundaries. A rectangular Cartesian coordinate system is adopted, so that the medium line of the channel begins at the center of the coordinate system. The boundaries of the stream are assumed to be given as $y=f(x) \pm h(x)$, where $f(x)$ and $h(x)$ are known

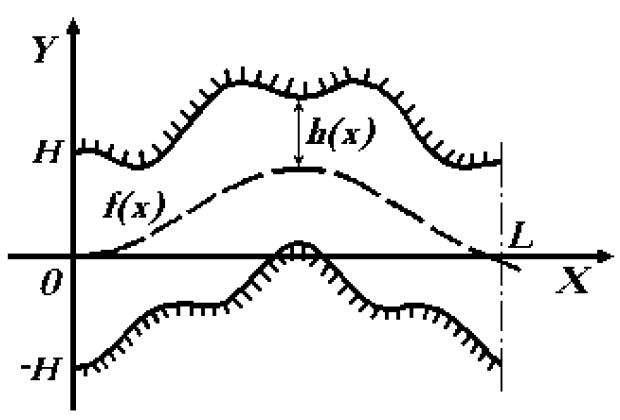

Figure 1. Disposition of the coordinate system.

functions.

Here, $f(x)$ defines the middle line of the channel and $h(x)$ defines the half-width one. We will consider the flow section $0 \leq x \leq L$, where $\mathrm{L}>>\max _{x \in[0, L]} h(x)$. Note that $f(0)=0$, because of the selected coordinate system. The governing equations for the admixture spreading in the 2D steady viscous fluid stream, which take into account diffusion and decay, are:

$$
\begin{aligned}
& u \frac{\partial u}{\partial x}+v \frac{\partial u}{\partial y}=-\frac{1}{\rho} \frac{\partial p}{\partial x}+\mu\left(\frac{\partial^{2} u}{\partial x^{2}}+\frac{\partial^{2} u}{\partial y^{2}}\right) \\
& u \frac{\partial v}{\partial x}+v \frac{\partial v}{\partial y}=-\frac{1}{\rho} \frac{\partial p}{\partial y}+\mu\left(\frac{\partial^{2} v}{\partial x^{2}}+\frac{\partial^{2} v}{\partial y^{2}}\right)
\end{aligned}
$$

$\frac{\partial u}{\partial x}+\frac{\partial v}{\partial y}=0$

$u=v=0 ; \quad(y=f(x) \pm h(x))$

$$
\int_{-h(0)}^{h(0)} \rho u(0, y) d y=Q
$$

$$
\frac{\partial c}{\partial t}+u \frac{\partial c}{\partial x}+v \frac{\partial c}{\partial y}=D\left(\frac{\partial^{2} c}{\partial x^{2}}+\frac{\partial^{2} c}{\partial y^{2}}\right)-\gamma c
$$

$$
\begin{aligned}
& \frac{\partial c}{\partial y}-\left(f^{\prime}(x)+h^{\prime}(x)\right) \frac{\partial c}{\partial x}=0 ; \\
& (y=f(x)+h(x))
\end{aligned}
$$




$$
\begin{aligned}
& \frac{\partial c}{\partial y}-\left(f^{\prime}(x)-h^{\prime}(x)\right) \frac{\partial c}{\partial x}=0 ; \\
& (y=f(x)-h(x)) \\
& c(x, y, 0)=a(x, y), \quad c(0, y, t)=b(y, t)
\end{aligned}
$$

After substitution of the above variables, equations (1)-(9) may be written out as follows (mark ' $\sim$ is omitted):

$$
\begin{aligned}
& \varepsilon u \frac{\partial u}{\partial x}+\varepsilon v \frac{\partial u}{\partial y}=-\frac{\partial p}{\partial x}+ \\
& +\operatorname{Re}^{-1}\left(\varepsilon^{2} \frac{\partial^{2} u}{\partial x^{2}}+\frac{\partial^{2} u}{\partial y^{2}}\right)
\end{aligned}
$$

The unknown functions are $u=u(x, y), v=v(x, y)$, and $c=c(x, y, t)$. They respectively denote the longitudinal and transverse components of the fluid velocity vector, the pressure and the concentration of the admixture. Furthermore, $\varrho$ is the constant density of the fluid, $\mu$ is the turbulent viscosity coefficient, $Q$ is the known water discharge at the section entry. Next, $D$ is the turbulent diffusion coefficient and $\gamma$ is the coefficient of the substance decay. Finally, the functions $a(x, y)$ and $b(x, y)$ set the initial contaminant distribution and the matter entering from the inlet section $x=0$, respectively.

The admixture is passive, so the liquid density, viscosity and velocity are independent from the substance concentration, the system of equations (1)(9) can be split in two parts. First, we solve the equations (1)-(5) to find the velocity field $(u(x, y)$, $v(x, y))$ and pressure $p(x, y)$. Then we solve equations (6)-(9) with known velocity and pressure to find the concentration $c(x, y, t)$.

\section{CONVERSION TO DIMENSIONLESS VARIABLES}

There are two spatial characteristic scales in the model, the length $L$, for the x-direction and the width $H=h(0)$, for the y-direction. They result from the problem when $L>>H$. Therefore, we introduce the parameter $\varepsilon=H / L$ and make use of the method proposed by Nadolin, Botchev and Nicolayev (1997).

Two velocity scales are used: $U=Q /(H \varrho)$, for the longitudal velocity and $V=\varepsilon U$ for the transversal one. $P=\varrho U^{2} L / H$ will be taken as the pressure scale, $T=L / U$ as the time scale and $\mathrm{C}=\max _{x, y} a(x, y)$

$$
\begin{aligned}
& \varepsilon^{3} u \frac{\partial v}{\partial x}+\varepsilon^{3} v \frac{\partial v}{\partial y}=-\frac{\partial p}{\partial y}+ \\
& +\operatorname{Re}^{-1} \varepsilon^{2}\left(\varepsilon^{2} \frac{\partial^{2} v}{\partial x^{2}}+\frac{\partial^{2} v}{\partial y^{2}}\right)
\end{aligned}
$$

$$
\frac{\partial u}{\partial x}+\frac{\partial v}{\partial y}=0
$$

$u=v=0 \quad(y=f(x) \pm h(x))$

$\int_{-1}^{1} u(0, y) d y=1$

$$
\begin{aligned}
& \frac{\partial c}{\partial t}+u \frac{\partial c}{\partial x}+v \frac{\partial c}{\partial y t}= \\
& =P e^{-1}\left(\varepsilon^{2} \frac{\partial^{2} c}{\partial x^{2}}+\frac{\partial^{2} c}{\partial y^{2}}\right)-\lambda c
\end{aligned}
$$

$$
\frac{\partial c}{\partial y}-\varepsilon^{2}\left(f^{\prime}(x)+h^{\prime}(x)\right) \frac{\partial c}{\partial x}=0
$$

$(y=f(x)+h(x))$

$$
\begin{aligned}
& \frac{\partial c}{\partial y}-\varepsilon^{2}\left(f^{\prime}(x)-h^{\prime}(x)\right) \frac{\partial c}{\partial x}=0 \\
& (y=f(x)-h(x))
\end{aligned}
$$

as the scale for the concentration. Thus, new dimensionless variables are introduced by formulae:

$$
\begin{aligned}
& x=L \tilde{x} ; y=H \tilde{y} ; t=T \tilde{t} ; u=U \tilde{u} ; v=V \tilde{v} \\
& p=P \tilde{p} ; c=C \tilde{c} ; a=C \tilde{a} ; b=C \tilde{b} ; \\
& f=H \tilde{f} ; h=H \tilde{h} .
\end{aligned}
$$

$$
c(x, y, 0)=a(x, y) ; \quad c(0, y, t)=b(y, t)
$$

The dimensionless parameters, the Reynolds number $R e$, the diffusion Peclet number $P e$ and the dissipation parameter $\lambda$ are defined as follows: 
$R e=\frac{H U}{\mu} ; P e=\frac{H V}{D} ; \lambda=\gamma T$

EQUATIONS OF MATHEMATICAL MODEL

Equations (10) - (18) are solved, by substituting the asymptotic $\varepsilon$-power expansion into the system and gathering the terms that do not contain the $\varepsilon$ yield:

$$
\begin{aligned}
& \frac{\partial^{2} u_{0}}{\partial y^{2}}=\operatorname{Re} \frac{\partial p_{0}}{\partial x} \\
& \frac{\partial p_{0}}{\partial y}=0 \\
& \frac{\partial u_{0}}{\partial x}+\frac{\partial v_{0}}{\partial y}=0
\end{aligned}
$$

$u_{0}=v_{0}=0 \quad(y=f(x) \pm h(x))$

$$
\int_{-1}^{1} u_{0}(0, y) d y=1
$$

$\frac{\partial c_{0}}{\partial t}+u_{0} \frac{\partial c_{0}}{\partial x}+v_{0} \frac{\partial c_{0}}{\partial y}=P e^{-1} \frac{\partial^{2} c_{0}}{\partial y^{2}}-\lambda c_{0}$

$\frac{\partial c_{0}}{\partial y}=0 \quad(y=f(x) \pm h(x))$

$$
c_{0}(x, y, 0)=a(x, y) ; \quad c_{0}(0, y, t)=b(y, t)
$$

Equations (20)-(27) will be referred as the initial approximation or the base mathematical model.

For the terms containing the first power of $\varepsilon$ we have:

$$
\begin{aligned}
& u_{0} \frac{\partial u_{0}}{\partial x}+v_{0} \frac{\partial u_{0}}{\partial y}=-\frac{\partial p_{1}}{\partial x}+R e^{-1} \frac{\partial^{2} u_{1}}{\partial y^{2}} \\
& \frac{\partial p_{1}}{\partial y}=0 \\
& \frac{\partial u_{1}}{\partial x}+\frac{\partial v_{1}}{\partial y}=0 \\
& u_{1}=v_{1}=0 \quad(y=f(x) \pm h(x))
\end{aligned}
$$

$$
\int_{-1}^{1} u_{1}(0, y) d y=0
$$

$$
\begin{aligned}
& \frac{\partial c_{1}}{\partial t}+u_{0} \frac{\partial c_{1}}{\partial x}+v_{0} \frac{\partial c_{1}}{\partial y}+u_{1} \frac{\partial c_{0}}{\partial x}+v_{1} \frac{\partial c_{0}}{\partial y}= \\
& =P e^{-1} \frac{\partial^{2} c_{1}}{\partial^{2} y}-\lambda c_{1} \\
& \frac{\partial c_{1}}{\partial y}=0 \quad(y=f(x) \pm h(x)) \\
& c_{1}(x, y, 0)=0 ; \quad c_{1}(0, y, t)=0
\end{aligned}
$$

Equations (28)-(35) constitute the correcting system.

\section{CONSIDERING OF THE BASE MODEL}

For the hydro-dynamical subsystem, equ. (20)(24) of the base model, an analytical solution can be obtained in the same way as for a long pipe (Kotorynski, 1994):

$$
u_{0}(x, y)=-\frac{3}{4 h^{3}(x)}\left[(y-f(x))^{2}-h^{2}(x)\right]
$$

$$
v_{0}(x, y)=-\frac{3}{4 h^{3}(x)}
$$

$$
\left[\frac{h^{\prime}(x)}{h(x)}(y-f(x))+f^{\prime}(x)\right]
$$

$$
\left[(y-f(x))^{2}-h^{2}(x)\right]
$$

$$
p_{0}^{\prime}(x)=-\frac{3}{2 \operatorname{Reh}^{3}(x)}
$$

For the straight channel, of constant width $(f(x)=$ 0 and $h(x)=1)$, the velocity transversal component (37) is equal to zero and formula (36) for the longitudal velocity gives the Poiseuille flow in pipe with a constant section.

Let us consider the base model equations, for the concentration given by equ. (25)-(27). To get a numerical solution, the flow domain is reduced to a rectangle by the substitution:

$x \rightarrow x, y \rightarrow(y-f(x)) / h(x)$ 
Then

$$
\begin{aligned}
\frac{\partial}{\partial x} & \rightarrow \frac{\partial}{\partial x}-\frac{f^{\prime}(x)+y h^{\prime}(x)}{h(x)} \frac{\partial}{\partial y}, \\
\frac{\partial}{\partial y} & \rightarrow \frac{1}{h(x)} \frac{\partial}{\partial y} \text { and } \frac{\partial^{2}}{\partial y} \rightarrow \frac{1}{h^{2}(x)} \frac{\partial^{2}}{\partial y}
\end{aligned}
$$

For the new variables, the following initialboundary value problem occurs, and it is solved numerically:

$$
\begin{aligned}
& \frac{\partial c_{0}}{\partial t}+u_{0} \frac{\partial c_{0}}{\partial x}=P e^{-1} \frac{\partial^{2} c_{0}}{\partial y^{2}}-\lambda c_{0} \\
& u_{0}(x, y)=-\frac{3}{4 h(x)}\left(y^{2}-1\right) \\
& \frac{\partial c_{0}}{\partial y}=0 \quad(y= \pm 1) \\
& c_{0}(x, y, 0)=\alpha(x, y) \\
& c_{0}(0, y, t)=b(x, t)
\end{aligned}
$$

\section{STUDY OF THE CORRECTING SYSTEM}

The analytical solution for the hydro-dynamical subsystem of equations (28)-(32) of the correcting system can be found after substitution of equations (36)-(38) into (28)-(32):

$$
\begin{aligned}
& u_{1}=\frac{9 \operatorname{Re}}{4 h^{3}(x)}\left\{\frac{3}{35} h^{\prime}(x)\left[(y-f(x))^{2}-h^{2}(x)\right]-\right. \\
& -\frac{h^{\prime}(x)}{4 h^{4}(x)}\left[\frac{(y-f(x))^{6}}{30}-\frac{(y-f(x))^{4}}{6} h^{2}(x)+(45)\right. \\
& \left.\left.+\frac{(y-f(x))^{2}}{2} h^{4}(x)-\frac{11}{30} h^{6}(x)\right]\right\}
\end{aligned}
$$$$
v_{1}=\frac{9 \operatorname{Re}}{4 h^{3}(x)}\left\{\left[f^{\prime}(x)+\frac{h^{\prime}(x)}{h(x)}(y-f(x))\right]\right.
$$$$
\left[\frac{3}{35} h^{\prime}(x)\left[(y-f(x))^{2}-h^{2}(x)\right]-\frac{h^{\prime}(x)}{4 h^{4}(x)}\right.
$$

$$
\left\lceil\frac{(y-f(x))^{6}}{30}-\frac{(y-f(x))^{4}}{6} h^{2}(x)+\right.
$$

$-\frac{(y-f(x))^{4}}{30} h^{2}(x)+\frac{11}{210}(y-f(x))^{2}$

$\left.\left.h^{4}(x)-\frac{h^{5}(x)}{42}\right]\right\}$

$p_{1}^{\prime}=\frac{27}{70} \frac{h^{\prime}(x)}{h^{3}(x)}$

For a channel of uniform width, $(\mathrm{h}(\mathrm{x})=1$ and $\left.\mathrm{h}^{\prime}(\mathrm{x})=0\right)$, the correction to velocity base field will be equal to zero.

For obtaining a correction after the change of variables (39) we have:

$$
\begin{aligned}
& \frac{\partial c_{1}}{\partial t}+u_{0} \frac{\partial c_{1}}{\partial x}-P e^{-1} \frac{\partial^{2} c_{1}}{\partial^{2} y}-\lambda c_{1}= \\
& =-u_{1} \frac{\partial c_{0}}{\partial x}-w_{1} \frac{\partial c_{0}}{\partial y}
\end{aligned}
$$

$u_{1}(x, y)=-\frac{3 \operatorname{Reh}^{\prime}(x)}{32 h(x)}$

$\left[\frac{y^{6}}{5}-y^{4}+\frac{33}{35} y^{2}-\frac{1}{7}\right]$

$w_{1}(x, y)=\frac{3 R e}{32} h^{\prime \prime}(x) y$

$\left[\frac{y^{6}}{35}-\frac{y^{4}}{5}+\frac{11 y^{2}}{35}-\frac{1}{7}\right]$

$\frac{\partial c_{1}}{\partial y}=0 \quad(y= \pm 1)$

$c_{1}(x, y, 0)=0 ; \quad c_{1}(0, y, t)=0$

The right part of the equation (18) is determined after solving the system (41)-(44). We note that 
for a channel of uniform width the system (48)(52) has a trivial solution $c_{1}(x, y, t)=0$.

\section{NUMERICAL TESTS}

We have made a series of numerical experiments on the basis of the proposed mathematical model. The propagation of a contaminant spot is examined. The initial and boundary conditions are determined by the functions:

$$
\begin{aligned}
& a(x, y)= \\
& \left\{\begin{array}{ll}
1-\frac{\left|x-x_{*}\right|}{l}, & \left|x-x_{*}\right| \leq l \\
0, & \left|x-x_{*}\right|>l
\end{array} \quad b(y, t)=0\right.
\end{aligned}
$$

The parameter $l$, where $0<l<<1$, characterizes the variation of a spot concentration across the longitudinal coordinate, and the parameter determines a position of the spot's centerline (Fig. 2).

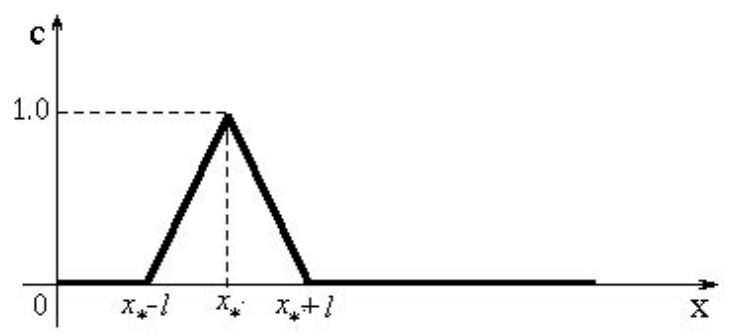

Figure 2. The initial distribution of contaminant (constant in y-direction)

The values $l=0.09, x_{*}=0.12$ were used for all calculations. The computational domain was $0 \leq x \leq$ $1.2 ;-1 \leq y \leq 1$.

Equations (41)-(44) and (48)-(52) were solved using a coupled finite element method and a finite difference method with bilinear rectangular elements in space and with Crank-Nicolson scheme in time.

We have carefully studied the influence of the parameter $P e$ on the spot spreading. The value of parameter $\lambda$ was 0.01 in all calculations. The results of numerical experiments are presented at the Fig. 3. The contaminant distribution is shown for different $P e$ at the final time $t=1$. The channel shape is $y= \pm(1+0.1 \sin 12 x)$, in dimensionless variables.

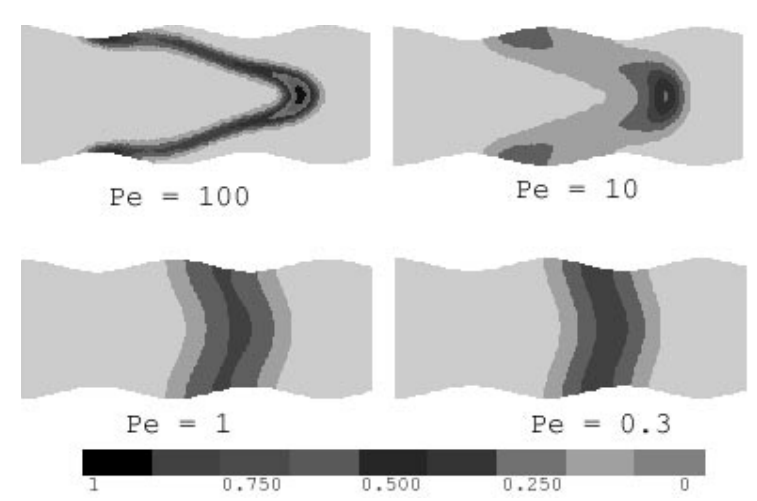

Figure 3. The concentration distribution for various values of $P e$.

\section{CONCLUSIONS}

In the present work, we introduced a distributed model for the advection process of a substance in a lengthy curvilinear channel (or river) 2D stream. A nice feature of the model is its simplicity: the hydrodynamical part of the model turns out to be integrable analytically, so that the problem is reduced to the non-steady truncated convection-diffusion problem. The model can be used as a part of the more comprehensive ecosystem models.

\section{REFERENCES}

Aris, R. (1956), On the dispersion of solute in a fluid flowing through a tube, Proc. Roy. Soc. London Ser. A, 235, 67-77.

Babayan, A.V. and Nadolin, K.A. (1997), Modelling contaminant spreading in 2D plane steady channel flow, In: Proceedings of VII All-Russian Conference "Modern problems in mathematical modelling", Novorossiysk, Rostov-on-Don State University, 15-21.

Chatwin, P.C. and Allen, C.M. (1985), Mathematical models of dispersion in rivers and estuaries, Ann. Rev. Fluid Mech. 17, 319-349.

Fife, P.C. and Nicholes, K.R. (1975), Dispersion in flow through small tubes, Proc. Roy. Soc. London Ser. A, 344, 131-145. 
Fisher, H.B. (1969), The effect of bends on dispersion in streams, Water Resource Research, 5, 496-506.

Kotorynski, W.P. (1994), Dispersion in pipes with slowly varying cross section, SIAM J. Math. Anal. 25, 915-940. Monin, A.S. and Yaglom, A.M. (1979), Statistical fluid mechanics, Vol. 1 Cambridge (Mass., USA), MIT Press. Nadolin, K.A., Botchev, M.A. and Nicolayev, I.A. (1998), Substance Advection by a Steady 2D Stream of the Viscous Fluid in a Lengthy Free-Surfaced Canal, In: Proceedings of the 2nd Int. Symp. on Turbulence, Heat and Mass Transfer, June 9-12, 1997, Delft, Netherlands, 409-418.

Rosencrans, S. (1997), Taylor dispersion in curved channels, SIAM J. Appl. Math. 57, 1216-1241.

Taylor, G.I. (1953), Dispersion of soluble matter in solvent flowing slowly through a tube, Proc. Roy. Soc. London Ser. A, 219, 186-203.

Taylor, G.I. (1954), The dispersion of matter in turbulent flow through a pipe, Proc. Roy. Soc. London Ser. A, 223, 446-468.

Yotsukura, N. and Sayre, W.W. (1976), Transverse moving in natural channels, Water Resources Research, 12, 695-704. 